\title{
Entanglement Percolation in Quantum Complex Networks
}

\author{
Martí Cuquet and John Calsamiglia \\ Grup de Física Teòrica, Universitat Autònoma de Barcelona, 08193 Bellaterra, Barcelona, Spain
}

(Received 16 June 2009; published 10 December 2009)

\begin{abstract}
Quantum networks are essential to quantum information distributed applications, and communicating over them is a key challenge. Complex networks have rich and intriguing properties, which are as yet unexplored in the quantum setting. Here, we study the effect of entanglement percolation as a means to establish long-distance entanglement between arbitrary nodes of quantum complex networks. We develop a theory to analytically study random graphs with arbitrary degree distribution and give exact results for some models. Our findings are in good agreement with numerical simulations and show that the proposed quantum strategies enhance the percolation threshold substantially. Simulations also show a clear enhancement in small-world and other real-world networks.
\end{abstract}

DOI: 10.1103/PhysRevLett.103.240503

PACS numbers: 03.67.Bg, 64.60.ah, 89.75.Hc

A broad variety of natural and socioeconomic phenomena such as protein-protein interactions, the brain, the power-grid, friendship networks, the Internet, and foodweb, can all be modeled by graphs, i.e., by sets of nodes and edges representing the relation between them. Complex networks $(\mathrm{CN})$ cover the wide range between regular lattices and completely random graphs. Their ubiquity has triggered an intense research activity on $\mathrm{CN}$ involving applied works, but also fundamental studies in theoretical physics and mathematics that aim at unveiling their underlying principles. Understanding structural properties of $\mathrm{CN}$ is very important as they crucially affect their functionality. For instance, the topology of a social network affects the spread of information or diseases, and the architecture of a computer network determines its robustness under router failures. The appearance of a giant connected component is a critical phenomenon related to percolation theory $[1,2]$. If, say, the infection probability of a disease exceeds a critical value, an outburst of the disease occurs infecting most of the population, whereas below the critical value only a vanishing amount of nodes is affected. This threshold and the size of the outburst are key quantities that strongly depend on the $\mathrm{CN}$ structure.

This Letter merges the field of $\mathrm{CN}$ with the also new and rapidly developing field of quantum information (QI). Networks already have a prominent role in QI, e.g., in the context of measurement-based quantum computation [3], in the characterization of graph states [4], and, more naturally, as the physical substrate of nodes and channels for multipartite quantum communication protocols. Such a "quantum internet" [5] supports QI applications that fill the technological gap between the already available bipartite applications like quantum key distribution, and the appealing but still remote quantum computer. The reason for studying quantum $\mathrm{CN}$, as opposed to regular lattices considered so far, is threefold. First, it is very plausible that future quantum communication networks acquire a complex topology resembling that of existing networks. This can certainly be the case if methods are developed to use current communication networks at a quantum regime, or if new quantum networks grow driven by similar sociopolitical mechanisms. Second, CN have nontrivial topological features not found in regular lattices, e.g., robustness to random errors, that can be useful in the design of novel quantum networks. Third, from a more theoretical perspective, although few qubit states have been successfully characterized, an understanding of multipartite entanglement of many-particle systems is still lacking. As we will show here, some quantities which are exceedingly hard to calculate for states defined over regular lattices can be carried with ease in $\mathrm{CN}$ (which are defined through statistical properties).

The goal of entanglement percolation [6] is to establish a maximally entangled state (which via quantum teleportation amounts to a perfect single-use quantum channel) between two arbitrary nodes of a network, where nodes are connected by partially entangled states. This can be easily achieved between two neighbor nodes since a partially entangled state can be converted into a maximally entangled state (for short singlet) with a probability that depends on the initial amount of entanglement. In 1D networks, a singlet can only be established between two remote nodes with a probability that decays exponentially with the distance between them [6]. In contrast, higher dimensional networks have an increasing number of possible connecting paths giving rise to a percolation effect, i.e., to the appearance of a cluster spanning a significant fraction $S$ of the network. Once a path of singlets is formed, a maximally entangled state can be established between the end-nodes by means of entanglement swapping. Thus, two arbitrary nodes can be connected by a singlet if both belong to the same cluster. For large networks, this happens with a probability $S^{2}$ independent of the distance between the nodes and of the size of the network [7], but that strongly depends on its topology. Acín et al. [6] found a type of lattice where the value of the percolation threshold, i.e., the 
required amount of initial entanglement, can be reduced by some quantum preprocessing: local quantum measurements are performed on some selected nodes of the honeycomb lattice turning it into a triangular with a lower threshold. In [8], other local strategies were studied in detail, and entanglement percolation was shown to appear in few more 2D lattices.

In this Letter, we study entanglement percolation in complex networks. We first derive general results for random networks with arbitrary uncorrelated degree distributions. From here, we can obtain exact values for the percolation threshold and the probability $S$. We next complement these results with numerical simulations on these and other paradigmatic $\mathrm{CN}$, with emphasis on those that mimic existing communication networks.

A network is naturally represented by a graph, which consists of $N$ vertices and a number of edges that connect some pairs of vertices. The degree of a vertex, $k$, is the number of edges connecting to it, and in general follows a probability distribution $P(k)$. A connected component, or cluster, is a subgraph where any two vertices are connected by at least one path and no more vertices can be added without losing this property. If edges are occupied with probability $p$, one can ask which is the distribution of connected component sizes when this probability varies. For low values of $p$, many small clusters exist. As $p$ increases, clusters start to grow and join each other. In general, the cluster size is vanishingly small compared to the size of the network, until a critical value of $p=p_{c}$ where a giant connected component (GCC) starts to span it: in the asymptotic limit, this is the only component of finite relative size $S>0$ for all $p \geq p_{c}$.

We consider quantum networks, as in Ref. [6], whose edges correspond to pairs of pure entangled states $|\psi\rangle^{\otimes 2}$, where $|\psi\rangle=\sqrt{\lambda_{0}}|00\rangle+\sqrt{\lambda_{1}}|11\rangle$ is a two qubit state with Schmidt coefficients $\sqrt{\lambda_{0}} \geq \sqrt{\lambda_{1}} \geq 0$. Each partially entangled state $|\psi\rangle$ can be converted into a singlet by LOCC with the singlet conversion probability (SCP) $p=$ $\min \left[1,2\left(1-\lambda_{0}\right)\right]$ [9]. If the two nodes share two copies of $|\psi\rangle$, the probability that at least one of them is converted into a singlet is, of course, $p_{2}=2 p-p^{2}$. However, they can do better since the largest Schmidt coefficient of $|\psi\rangle^{\otimes 2}$ is $\lambda_{0}$, and hence its optimal SCP is $p_{2}=\min [1,2(1-$ $\left.\left.\lambda_{0}^{2}\right)\right]=\min \left(1,2 p-p^{2} / 2\right)$. From here on, "with" and "without distillation" respectively mean that the optimal or the former sequential transformation is used. Applying the above singlet conversion to each edge of the network is called classical entanglement percolation [6]. As already mentioned, a new quantum feature appears when we allow for other local quantum operations that transform the network geometry and thereby change the percolation threshold. The basic ingredient is entanglement swapping (or "swap") illustrated in Fig. 1. The party at a central node $c$ performs a Bell measurement on two qubits, each of them belonging to states $|\psi\rangle$ shared with different nodes ( $a$ and $b)$. After this operation, the central qubits become disen- tangled from $a$ and $b$, but in return, an entangled state is established between $a$ and $b$, which on average has the same SCP as $|\psi\rangle$. Note, however, that this operation cannot be repeated using newborn edges since they are not in state $|\psi\rangle$. The " $q$-swap" operation that we use here performs the "swap" transformation between successive pairs of neighbors of a central node of degree $q$, effectively changing the initial $q$-star into a $q$-cycle (Fig. 1). These $q$-swaps are made on nodes of certain degrees, depending on the network structure, with the only condition that no operation is done on neighboring sites. Thus, the strategy uses only local information of the network: the degree of the target node and the status of its neighbors.

We now proceed to present our results for general random graphs of arbitrary (uncorrelated) degree distribution. We use the generating function formalism [10,11]. We begin by defining the generating function of the degree distribution by $G_{0}(x)=\sum_{k=0}^{\infty} P(k) x^{k}$. Since $P(k)$ is a probability, $G_{0}$ is well normalized, $G_{0}(1)=1$, and convergent for $|x| \leq 1$. What makes generating functions especially suited here is that it is straightforward to convolute probability distributions; e.g., $G_{0}(x)^{m}$ generates the probability that $m$ arbitrary nodes have a total of $k$ edges leading to them. The key probability distribution in percolation is that of reaching a finite component of size $s$ when following a random edge to one of its ends, generated by $H_{1}(x)$. The size is zero when the edge is not occupied. With probability $p_{2}$ it is occupied, and the discovered vertex has degree distribution $P_{1}(k)=k P(k) /\langle k\rangle$ generated by $\sum_{k=0}^{\infty} P_{1}(k) x^{k}=x \frac{G_{0}^{\prime}(x)}{G_{0}^{\prime}(1)} \equiv x G_{1}(x)$. Thus, $s$ is one plus the size of the new $k-1$ reachable components. The size of each of them is again generated by $H_{1}(x)$, and their sum by $\sum_{k=0}^{\infty} P_{1}(k)\left[H_{1}(x)\right]^{k-1}$. Therefore, $H_{1}(x)$ fulfills the consistency equation

$$
H_{1}(x)=\left(1-p_{2}\right)+p_{2} x G_{1}\left(H_{1}(x)\right) .
$$

It is crucial to notice that by restricting to finite $s$, we have explicitly excluded the infinite GCC from $H_{1}(x)$ [12]. The total probability that an edge connects to a finite component is $u \equiv H_{1}(1)$, and due to (1) satisfies $u=1-$ $p_{2}+p_{2} G_{1}(u)$. Below the critical $p<p_{c}$, there is a unique solution $u=1$, while at $p \geq p_{c}$, a new solution $u<1$ appears. Hence, the critical probability is the smallest value of $p$ for which the second solution appears.

We can similarly compute the function $H_{0}(x)$ generating the probability that a randomly chosen vertex belongs to a

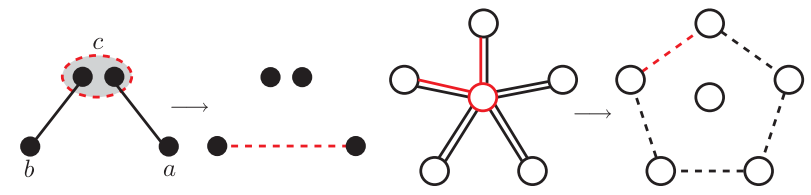

FIG. 1 (color online). Entanglement swapping (left) and 5swap transformation (right). Dots represent qubits, circles nodes, solid lines partially entangled states $|\psi\rangle$, and dashed lines correspond to states with the same SCP as $|\psi\rangle$. 
finite component of size $s$. Since $k$ edges emerge from this vertex with probability $P(k)$, we find $H_{0}(x)=x G_{0}\left[H_{1}(x)\right]$. The probability that a randomly chosen vertex belongs to the GCC is $S=1-H_{0}(1)=1-G_{0}(u)$. From $H_{0}(x)$, we can compute many other relevant properties, e.g., the mean component size below $p_{c},\langle s\rangle=H_{0}^{\prime}(1)$, which behaves like a susceptibility diverging at the phase transition.

Our goal now is to understand how quantum operations change the percolation properties of the original network. Every particular $q$-swap can be implemented (or not) with probability $\Pi_{q}$ (or $1-\Pi_{q}$ ) on nodes of degree $q$. Giving the values for each $\Pi_{q}$ specifies the quantum strategy. Now, instead of arriving to a vertex of degree $q$ connecting to other $q-1$ components, after a $q$-swap operation we arrive to a cycle of $q$ nodes (including the one we are coming from) connected via links with SCP equal to $p$ [13]. The size of such cycle and its emerging components is the sum of the probabilities that a connected string of $l$ vertices in the cycle is reached, multiplied by the generating function of the emerging component sizes:

$$
\begin{aligned}
C_{q}(x)= & \sum_{l=0}^{q-2}(l+1) p^{l}(1-p)^{2}\left\{x G_{1}\left[\tilde{H}_{1}(x)\right]\right\}^{l} \\
& +\left[q p^{q-1}(1-p)+p^{q}\right]\left\{x G_{1}\left[\tilde{H}_{1}(x)\right]\right\}^{q-1} .
\end{aligned}
$$

Therefore, the new $\tilde{H}_{1}(x)$ is of the same form of Eq. (1) plus a term $\tilde{H}_{1, q}(x)$ for each $q$-swap:

$$
\begin{gathered}
\tilde{H}_{1}(x)=1-p_{2}+p_{2} x G_{1}\left[\tilde{H}_{1}(x)\right]+\sum_{q \geq 2} \Pi_{q} \tilde{H}_{1, q}(x) \\
\tilde{H}_{1, q}(x)=P_{1}(q)\left\{\left(p_{2}-1\right)-p_{2} x\left[\tilde{H}_{1}(x)\right]^{q-1}+C_{q}(x)\right\} .
\end{gathered}
$$

At this stage, we can already calculate $\tilde{p}_{c}$ as the value of $p$ for which there exists a solution $0<u=\tilde{H}_{1}(1)<1$ to (3) at $x=1$. It is easy to see that each separate contribution $\tilde{H}_{1, q}(1)$ either increases or lowers the threshold, and thus for the optimal strategy each $\Pi_{q}$ is either 0 or 1 .

In order to calculate the relative size of the giant component $\tilde{S}$, we need to find the new $\tilde{H}_{0}(x)$. Given a vertex $q$, there is a probability $\eta_{q}$ that a $q$-swap can be performed on it, changing its degree from $q$ to zero and hence

$$
\tilde{H}_{0}(x)=x G_{0}\left[\tilde{H}_{1}(x)\right]+x \sum_{q \geq 2} \Pi_{q} \eta_{q} P(q)\left\{1-\left[\tilde{H}_{1}(x)\right]^{q}\right\} .
$$

From here, the order parameter $\tilde{S}=1-\tilde{H}_{0}(1)$ can be readily obtained by plugging in the solution of $\tilde{H}_{1}(1)=$ $u$. The probability $\eta_{q}$ that no other $q^{\prime}$-swap was performed in its neighbors can be computed up to any order in $p$ exactly [14]. When only one type of $q$-swap is performed, a compact, approximate expression exists $\eta_{q} \approx[1+$ $\left.\frac{q}{2} P_{1}(q)\right]^{-1}$. In the following, we study entanglement percolation enhancement in particular $\mathrm{CN}$ models.

The Bethe lattice is an infinite regular tree where every vertex has the same degree. Inserting $G_{0}(x)=x^{q}$ and $G_{1}(x)=x^{q-1}$ into (1) and (3) and solving the point at which the solution $u<1$ appears gives $(q-1)^{-1}=p_{2}$ and $\quad(q-1)^{-1}=(1-p)^{-1}\left\{2 p+p^{q}[p(q-1)-(q+1)]\right\}$ after $q$-swap is applied. Therefore, $q$-swap gives always a better threshold except for an infinite 1D chain $(q=2)$.

Erdös-Rényi (ER) graphs are maximally random graphs with the only constraint $\langle k\rangle=z$. An ER network has $N$ vertices, and each pair of them holds an edge with probability $z / N$. The degrees follow a Poisson distribution and $G_{0}(x)=G_{1}(x)=\exp [z(x-1)]$. The original threshold is given by $p_{2}=1 / z$. After, e.g., the 2 -swap and 3-swap operations, the thresholds are, respectively, $1 / z=$ $p_{2}+e^{-z}\left[-p_{2}+z\left(2 p-p^{2}\right)\right] \quad$ and $\quad 1 / z=p_{2}+$ $z e^{-z}\left[-p_{2}+z\left(1+p-p^{2}\right)\right]$. In general, the performance of different $q$-swaps depends on the mean degree $z$. Figure 2(a) shows the gain $\gamma=\left(\tilde{p}_{c}-p_{c}\right) / p_{c}$. The threshold can improve by a $20 \%$ ( $24 \%$ without distillation), compared to the $3 \%$ ( $16 \%$ without distillation) of the honeycomb-triangular transformation in [6]. The size of the GCC can be calculated exactly from the above results [14] and is plotted in Fig. 2(b) showing perfect agreement with numerical simulations. Note that $\tilde{S}$ attains smaller values for the transformed network even at $p=1$ due to the target nodes that become detached after the $q$-swap. Since one can always choose where to start applying the $q$-swaps, the probability of connecting two arbitrary nodes is actually $\hat{S}^{2}$, where $\hat{S}=\tilde{S} S_{1} / \tilde{S}_{1}$ is the probability that a node belongs to the GCC excluding the detached nodes and $S_{1}$ is the GCC size at $p=1$.

Scale-free networks: Real-world networks are not Poissonian but typically have a power-law (scale-free) degree distribution. Such networks have a GCC at all $p$, and a threshold appears only in scenarios where there is a cutoff at high degrees due to, e.g., targeted attacks, physical constraints or saturation effects. We have taken an exponential cutoff, $P(k)=C k^{-\tau} e^{-k / \kappa}$, and find once again
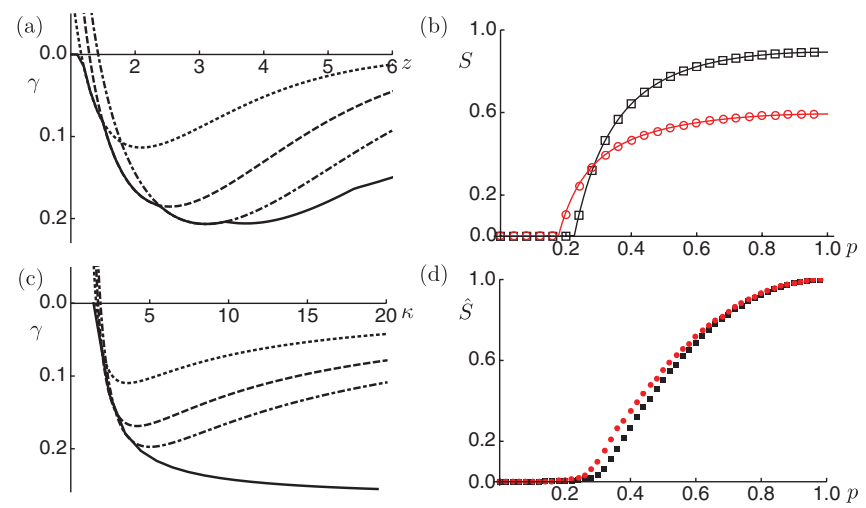

FIG. 2 (color online). (a) Gain $\gamma$ in the ER model as a function of the mean degree $z$ after 2-swap (dotted line), 2,3-swap (dashed line), 2,3,4-swap (dot-dashed line) and optimal $q$-swaps (solid line). (b) Size of the GCC as a function of $p$ in the $z=2.5$ ER network without distillation before (squares) and after 2,3-swap (circles). Points are simulation results for $N=$ $10^{6}$. (c) Same as (a) for scale-free model with $\tau=1$ as a function of $\kappa$. (d) Same as (b) for $W W W$ [15] with cutoff $k=$ $15\left(N \sim 10^{4}\right)$. 

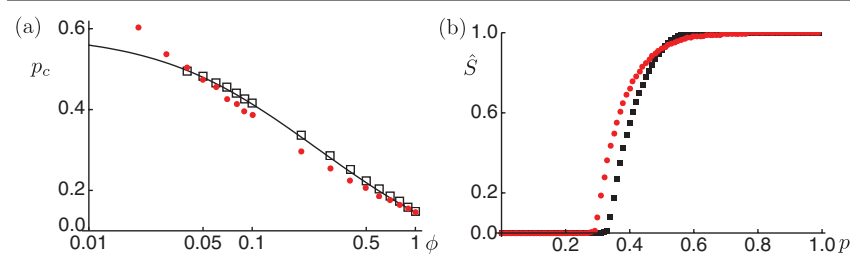

FIG. 3 (color online). Numerical results for the SW model with 2-swap and distillation. (a) $p_{c}$ as a function of $\phi$ before (squares) and after (disk) operations. Solid line is the classical analytic solution from [11]. (b) Size of the GCC as a function of $p$ before (squares) and after (circles) operations for $\phi=0.25$.

a significant improvement in $p_{c}$, up to $25 \%$ with distillation [Fig. 2(c)]. We have performed simulations for a realworld $W W W$ network [15], where we have introduced a cutoff neglecting nodes with $k \geq 15$ and, despite the finitesize effects, we clearly see a decrease in $p_{c}$ [Fig. 2(d)].

A Small World (SW) [16] is a network with ordered local structure and high level of clustering but still with surprisingly low average path length. We consider an SW model generated by placing $N$ vertices in a 1D chain. Then $N$ additional random edges, "shortcuts," are added with probability $\phi$. In this case, degrees are strongly correlated, and thus the above analysis does not apply. Still, simulations show that $q$-swaps can decrease $p_{c}$ (Fig. 3).

To summarize, we have studied entanglement percolation in complex networks-which are defined through their statistical properties and whose exact structure is not necessarily known - and shown that by a strategy that only uses local information, we can decrease the percolation threshold substantially (in some cases almost 1 order of magnitude more than found on the honeycomb lattice). We have exactly solved models of random graphs with arbitrary uncorrelated degree distribution. Moreover, we have numerically explored the behavior in SW networks and the $W W W$. In all cases, we find that entanglement percolation, far from being restricted to particular networks, is a quite general feature. Entanglement percolation is interesting from the discipline of $\mathrm{CN}$ since quantum mechanics introduces a new paradigm where networks can suffer nontrivial structural changes. We believe our results open prospects for many interesting synergies between the fields of quantum information and complex networks.

An essential issue for entanglement percolation schemes is their stability under noise. For amplitude damping, one can adapt the prescription in [17]. More general scenarios might require schemes that, e.g., use multipartite entanglement, use global knowledge of the network, concentrate different paths in a single one, or allow for the generation of new (imperfect) bonds during the protocol. Finally, note that $\mathrm{CN}$ can enjoy much better stability than lattices. In particular, in many $\mathrm{CN}$, the average path length scales with the size of the network as $\log N$, as opposed to $N^{1 / d}$ for $d$-dimensional lattices. Thus, the probability of establish- ing entanglement between two arbitrary nodes has a linear falloff, as opposed to a fatal exponential decay, and hence is non-negligible in large, but finite, networks.

We are grateful to M. Boguñá and A. V. Goltsev for discussions. We acknowledge financial support from the Spanish MICINN, through the Ramón y Cajal (J.C.) and AP2008-03048 (M.C.) and projects FIS2008-01236 and QOIT (CONSOLIDER2006-00019), and the Generalitat de Catalunya Contract No. 2009SGR985.

Note added.-Soon after the completion of this work, in [18] Perseguers et al. showed radically different properties between quantum and classical Erdős-Rényi graphs, and also stressed the interest in quantum $\mathrm{CN}$. Interesting results for entanglement distribution in regular lattices have also been recently reported in [19].

[1] G. Grimmett, Percolation (Springer-Verlag, Berlin, 1989).

[2] S. N. Dorogovtsev, A. V. Goltsev, and J. F. F. Mendes, Rev. Mod. Phys. 80, 1275 (2008).

[3] R. Raussendorf and H. J. Briegel, Phys. Rev. Lett. 86, 5188 (2001).

[4] M. Hein, J. Eisert, and H. J. Briegel, Phys. Rev. A 69, 062311 (2004).

[5] H. J. Kimble, Nature (London) 453, 1023 (2008).

[6] A. Acin, J. I. Cirac, and M. Lewenstein, Nature Phys. 3, 256 (2007).

[7] J. Calsamiglia et al., Phys. Rev. Lett. 95, 180502 (2005); L. Hartmann et al., J. Phys. B 40, S1 (2007); K. Kieling, T. Rudolph, and J. Eisert, Phys. Rev. Lett. 99, 130501 (2007); D. E. Browne et al., New J. Phys. 10, 023010 (2008).

[8] S. Perseguers et al., Phys. Rev. A 77, 022308 (2008); J. Lapeyre, J. Wehr, and M. Lewenstein, Phys. Rev. A 79, 042324 (2009).

[9] G. Vidal, Phys. Rev. Lett. 83, 1046 (1999).

[10] D. S. Callaway et al., Phys. Rev. Lett. 85, 5468 (2000); M. A. Serrano and M. Boguna, Phys. Rev. Lett. 97, 088701 (2006).

[11] C. Moore and M.E. J. Newman, Phys. Rev. E 62, 7059 (2000).

[12] We have assumed that components are treelike, since an edge exiting a finite component of size $s$ will connect back to the same component with a probability proportional to $s / N \rightarrow 0$. This obviously does not hold for the GCC.

[13] $q$-swaps introduce cycles, and components are no longer treelike. However, such cycles do not overlap and can be considered blocks of a treelike component.

[14] M. Cuquet and J. Calsamiglia (to be published).

[15] R. Albert, H. Jeong, and A. Barabási, Nature (London) 401, 130 (1999).

[16] D. J. Watts and S. H. Strogatz, Nature (London) 393, 440 (1998).

[17] S. Broadfoot, U. Dorner, and D. Jaksch, arXiv:0906.16221v2.

[18] S. Perseguers et al., arXiv:0907.3283v1.

[19] S. Perseguers, arXiv:0910.1459; S. Perseguers et al., arXiv:0910.2438. 